\title{
Potential Factors for Recurrence of Cholesteatoma in Children
}

\section{Ivan Baljosevic ${ }^{1 *}$, Aleksandar Sovtic ${ }^{2}$, Mladen Novkovic ${ }^{1}$ and Stefan Popovic $^{1}$}

${ }^{1}$ Department of Otorhinolaryngology, Mother and Child Health Institute-Belgrade, Faculty of Dentistry, Pancevo, Serbia

${ }^{2}$ Department of Pulmology, Mother and Child Health Institute-Belgrade, Medical faculty, Belgrade, Serbia

*Corresponding Author: Ivan Baljosevic, Department of Otorhinolaryngology, Mother and Child Health Institute-Belgrade, Faculty of Dentistry, Pancevo, Serbia.
Received: October 18, 2021

Published: November 30, 2021

(C) All rights are reserved by Ivan

Baljosevic., et al.

\begin{abstract}
Objective: Cholesteatoma of middle ear in children has a more aggressive development process and faster growth than that among adults. The aim of this study was to establish the dominant causes of cholesteatoma recurrence in children and to determine the optimal surgical treatment.

Methods: This study describes 92 cases of cholesteatomas found on 89 children. The first group consists of 44 cases of cholesteatomas that were operated with the wall up surgical technique, and the second group consists of 48 cases operated with the wall down surgical technique.

Results: Recurrence cholesteatoma was diagnosed in 18 (19.5\%) patients. In cases where the wall up, surgical technique was applied cholesteatoma recurrence was diagnosed in 14 (32\%) cases. By contrast, in the wall down surgical technique recurrence was found in only $4(8 \%)$.

Conclusion: Factors that can influence the appearance of recurrence are the: patient's age, type of applied surgical technique, size of mastoid process, degree of damage on the hearing chain and pathological changes in the second ear.

Keywords: Cholesteatoma; Children; Surgical Procedures; Recurrence
\end{abstract}

\section{Introduction}

Cholesteatoma represents a benign formation of epidermal origin and is formed by the accumulation of keratinizing squamous epithelium in the middle ear or other pneumatic spaces of the temporal bone. Aggressive-destructive growth causes bone resorption, destruction of the auditory chain and eardrum, with consequent hearing loss, vestibular dysfunction, facial paralysis, and intracranial complications. Previous studies have shown that cholesteatoma in children characterized more common recurrence due to anatomic and physiologic differences [1]. Eustachian tube anatomy and dysfunction predisposes children to more frequent infections and retraction pockets. Well pneumatized mastoids in children make them highly prone to disease compared to adults with more sclerotic mastoid bones. Most authors regard choles- teatoma in children to be more aggressive, with a higher proliferative activity and a higher rate of recurrence [2]. According to the literature, the percentage of recurrence is significantly higher in children younger than 8 , where occurrence is at $47 \%$, compared with the $24 \%$ in older children [3]. Thus, it is imperative to carefully choose the surgical intervention by which cholesteatoma is being removed.

The aim of this study is to establish the dominant causes of cholesteatoma recurrence in children and to determine the optimal surgical treatment.

\section{Material and Methods}

A prospective clinical study was conducted at Department of Otorhinolaryngology, Mother and Child Health Institute of Serbia, 
tertiary institution for treating children. We selected 92 cases of cholesteatoma which were surgically treated in 89 children. Three patients had bilateral cholesteatoma. The children were between 2 to 16 years old. The research was conducted from July 2009. to July 2018.

The inclusion criterion for follow-up was patients with COM (chronic suppurative otitis media) with cholesteatoma diagnosed after Otmicroscopy and CT scan. Exclusion criteria were children with dry perforation of tympanic membrane and tub tympanic COM.

The patient divided in two groups. The first group had 44 cases of cholesteatoma treated with canal wall up (CWU) technique. The second group had 48 cases of cholesteatoma treated with canal wall down (CWD) technique. The criterions for the application of canal wall down technique were the extension of cholesteatoma within the pneumatic spaces of the middle ear, damage of ossicular chain and destruction of bony walls. With the CWD technique, we performed reconstruction of the tympanic membrane and part of the posterior wall of the hearing canal, and reconstruction of ossicular chain, using the fascia of temporal muscle or tragal cartilage. During the surgical intervention, we diagnosed the extent of the cholesteatoma process. Localization was performed according to Slangerup [4], where the middle ear is divided into 4 localities (attic, tympanic cavity, antrum, and mastoid process).

The first postoperative control examination was performed seven days after the hospitalization, and then every 30 days during the first three months after the completion of treatment was. Control examinations were performed every three months for a period of one year. Children in both groups were monitored for at least one year after the treatment (Chart 1).

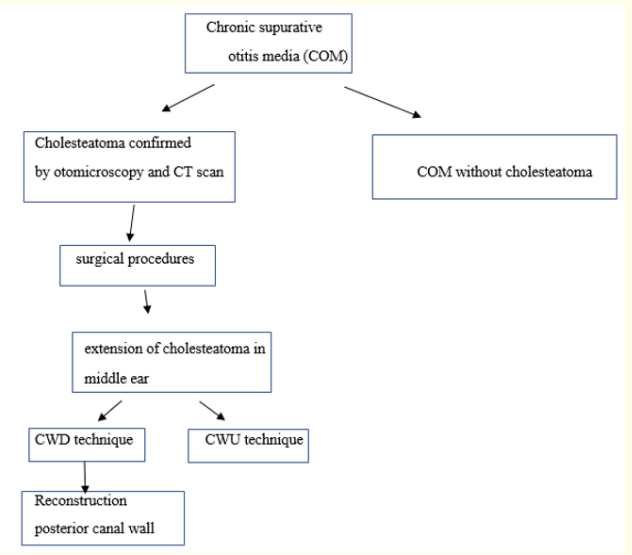

\section{Statistical analyses and methods}

Data were tabulated and analysed using the SPSS software program. Descriptive statistical methods, relative numbers, and methods for testing statistical hypotheses were used to analyse the primary data. Of the descriptive statistical methods, measures of central tendency (arithmetic mean and median), measures of variability (standard deviation) were used. Of the relative numbers, structure indicators expressed as percentages were used. In the process of testing statistical hypotheses, the chi-square test, Fisher's exact probability test, sign test, Mann-Whitney test and t-test were used. In the process of testing statistical hypotheses, 0.05 was selected for the significance level (alpha level).

\section{Results}

The recurrence of cholesteatoma was observed in 18 (19,5\%) cases. Recurrence was diagnosed in 14 (32\%) cases that had the canal wall up technique. In cases where we first applied canal wall down surgical technique, only 4 (8\%) patients had recurrence. Five $(30,6 \%)$ patients were not primarily operated in our institution. Four of the patients were treated using wall up technique and one underwent surgery with the wall down technique. The frequency of cholesteatoma recurrence is significantly higher in children surgically treated using the wall up technique $(p=0.005)$.

During the first surgical intervention, the most frequently diagnosed was extended cholesteatoma. In 47 (51\%) cases cholesteatoma was in the tympanic cavity and in mastoid process. The extent of cholesteatoma in the mastoid process was statistically higher in patients treated with the CWD technique $(\mathrm{p}<0.001)$.

Recurrence of cholesteatoma was diagnosed in 14 (77\% of the 18 patients with recurrence) children on the first year after the surgical intervention. The frequency of cholesteatoma recurrence was significantly higher in children treated with the wall up surgical technique $(\mathrm{p}<0.001)$. The shortest period in which we have diagnosed recurrence was 5 months after the first surgery. Among children surgically treated with CWU, 13 had recurrence, whereas in the CWD, only one child had recurrence. In children treated with the CWD technique, cholesteatoma recurrence was found to occur at a smaller percentage after a longer period (over 18 months with 3 out of 4 children). The incidence recurrence of cholesteatoma three years after the surgery was significantly higher for children treated with the wall up technique $(14 / 44=32 \%)$ compared with to those treated with the wall down technique $(4 / 48=8 \%)(\mathrm{p}=$ $0.005)$.

\section{Chart 1}


The average age of patients, with recurrence after the first surgery, in our groups was 8,6 (SD = 3,8). During the second surgical intervention, the most frequently diagnosed was extended cholesteatoma, which infected all pneumatic areas of the middle ear in 6 (42\%) children surgically treated with the CWU technique and in 3 (75\%) who were treated with the CWD technique. There is no statistically significant difference between the wall up and wall down surgical techniques according to the localization of cholesteatoma recurrence $(p=0.58)$. Furthermore, there is no statistically significant difference between the different localizations and the spread of primary cholesteatoma according to frequency of cholesteatoma recurrence $(p=0.47)$.

Damage of ossicular chain was found in 73 (79\%) of patients. The highest number of cholesteatoma recurrence was recorded in the group with a high level of ossicular chain damage. Eight percent of recidivism was recorded in the group with only partial damage and twenty-eight percent in the group with total absence of one hearing bone. There is statistically significant difference between children with a high level of ossicular chain damage and children with damaged hearing chain according to the frequency of cholesteatoma recurrence $(p=0.03)$.

Apparently, the development of cholesteatoma recurrence in the operated ear relates to pathological changes diagnosed in the second ear. The largest percentage of recurrence was in the cases where otitis media with effusion had been diagnosed, 4 (66\%) in the second ear. This could be explained by the poor function of Eustachian tube as well as poor ventilation on both ears. These may have compromised the success of the surgical treatment, which led to the development of recurrence. The frequency of cholesteatoma recurrence within three years after the surgery is significantly higher in children with pathological conditions in the second ear than those with normal conditions in second ear $(p=0.048)$.

The highest degree of cholesteatoma recurrence, in our study, was $29 \%$, recorded in the group with the small mastoid cavity. As mentioned earlier, this can be attributed to poor ear ventilation. The frequency of cholesteatoma recurrence within three years after surgery is significantly higher in children with undeveloped mastoid pneumatization than those with developed mastoid pneumatization $(\mathrm{p}=0.02)$ (Table 1 , Table 2).

\begin{tabular}{|l|c|}
\hline Variable & Statistic \\
\hline Sex, n (\%) Male Female & $60(65)$ \\
& $32(35)$ \\
\hline Age (year), mean (SD) & $9.9(3.6)$ \\
\hline Extension of cholesteatoma, n (\%) Attic + antrum & $18(20)$ \\
Attic + antrum + mastoid process & $27(29)$ \\
\hline Damage of hearing chain, median (range) & $47(51)$ \\
\hline Condition on opposite ear, n (\%) & $2(0-6)$ \\
\hline Normal Adhesive otitis Secretory otitis & $68(74)$ \\
Cholesteatoma & $11(12)$ \\
& $6(6)$ \\
\hline Pneumatization of mastoid process, n (\%) & $45(49)$ \\
\hline UndevelopedDeveloped & $47(51)$ \\
\hline Type of surgical intervention, n (\%) CWU CWD & $44(48)$ \\
& $48(52)$ \\
\hline
\end{tabular}

Table 1: Demographic and clinical characteristics of study patients.

\begin{tabular}{|l|c|c|}
\hline & Beta & P \\
\hline Age & -0.15 & 0.039 \\
\hline Condition on opposite ear & Reference category & 0.022 \\
Normal Adhesive otitis & 1.43 & 0.005 \\
Secretory otitis Cholesteatoma & 1.96 & 0.007 \\
& 1.68 & \\
\hline Pneumatization & 1.04 & 0.029 \\
\hline Damage of hearing chain & 1.37 & $<0.001$ \\
\hline
\end{tabular}

Table 2: Time to recurrence analysis-Cox regression model.

\section{Discussion}

It was found that the recurrence of pathological process, is more frequently detected in cases treated with the CWU surgical technique. Schmid., et al. [5], found that cholesteatoma in children is twice more likely to appear if the surgeon applies the closed technique than open technique. Chin sky reported that recurrence was present in $12 \%$ of patients that had the CWD technique and in 35\% with CWU technique [6]. According to Scharff and Strascnik, cholesteatoma in children is more aggressive compared to that in adults. They found that the total percentage of recurrence is $16 \%$, and with closed technique - $17 \%$ and with open technique - $12 \%$

Citation: Ivan Baljosevic., et al. "Potential Factors for Recurrence of Cholesteatoma in Children". Acta Scientific Orthopaedics 4.12 (2021): 53-58. 
[1]. Single-stage canal wall up mastoidectomy was significantly associated with a higher risk of cholesteatoma recidivism compared to canal wall down technique in children with acquired cholesteatoma [7]. Khavasi., et al. [8] suggested that canal wall down mastoidectomy is the optimal management technique for adequate exposure and removal of cholesteatoma in pediatric population.

Soldati and Mudry [9] compared the number of revision operations with the number of recurrences that appeared in operated patients. They found 46\% recurrence of cholesteatoma in the CWU technique and $40 \%$ in the CWD technique. Piras., et al. [10] also found that the percentage of recurrence in the CWU technique was significantly higher than in the CWD technique $(22,9 \%$ vs $2,3 \%)$. Darrouzet., et al. note that the wall down technique does not exclude residual lesion (23.8\% in their study) [11[. They also stated that there is $30 \%$ to $38 \%$ residual after the wall down technique is carried out, with temporal muscle flap and mastoid obliteration. In our study, cholesteatoma recurrence was diagnosed in 18 (19.5\%) patients. In cases where the wall, up technique was applied recurrence was diagnosed in 14 (32\%) cases. Out of the 18 cases in our study, cholesteatoma recurrence was diagnosed within the first year after surgery in 14 (77\%) cases: that is, in the13 cases where we applied the wall up technique and in single case with the wall down technique. We therefore conclude that, most likely, cholesteatoma was not completely removed during the first surgery.

Slangerup, Deresiewicz and Tos [4] found 47\% recurrence in children younger than 8 years compared with $24 \%$ of children over 8. They attributed this to the more aggressive nature of cholesteatoma in early childhood. They also found that this group had poor ear ventilation and the highest degree of hearing chain damage. In our study the average age of children with recidivism after the first operation was 8,6 years. Other authors also show in their studies that the degree of recurrent cholesteatoma is higher in younger children [12-14]. Ilno., et al. [15] reports that in children younger than ten-25\% had recurrent [recidivism of cholesteatoma and $42 \%$ had residual cholesteatoma, where tympanoplasty was performed with reconstruction of the posterior wall. As mastoid development in young children is still not completed, they become prone to more frequent inflammation of the middle ear and are more likely to develop infection of upper respiratory airways which causes Eustachian tube dysfunction.

Some authors believe that there is a connection between the degree of damage of the hearing chain and cholesteatoma recur- rence. Roger., et al. [16] reported 4 factors that are connected to cholesteatoma recurrence in children: compromising of back parts of middle ear, erosion of hearing chain, surgeon's skill and assumption of incomplete removal. Rosenfeld., et al. described the condition of 244 operated ears at 232 children. They found a connection between recurrence and the erosion of ossicular chain, but not with the applied surgical technique. A greater number of infected bones increases the risk of recurrence [17]. In our research, there is statistically significant difference between children with a high level of hearing chain damage and children with damaged hearing chain according to the frequency of cholesteatoma recurrence $(\mathrm{p}$ $=0.33)$.

Surgeon experience is also crucial in the conduct of surgical techniques. We have noted that out of a total number of cases with recurrence (18), one patient with the wall down technique and 4 with the wall up technique, were not primarily operated in our institution. These $5(36 \%)$ patients were operated on in the secondary type of medical institutions. There was no need for a third surgery in any of the cases. Existing literature considers the experience of the surgeon as an important factor in the appearance of the cholesteatoma recurrence. Soldati and Mudry [9] described 48 cases of cholesteatoma, where upon second examination, 22 cases (46\%) had cholesteatoma. On the third examination, 8 (44\%) of them had a recurrence of cholesteatoma. To completely address the condition, 4 ears were operated 4 times. Fageeh., et al. [18] also state that cholesteatoma recurrence in majority of the cases developed within the sinus tympani, facial recesses, and frontal tympani cells. They assume that cause was the inadequate and erroneous selection of surgical technique. In an earlier study, the frequency of recidivism in the wall up technique was found to be $39 \%$. The development of cholesteatoma recurrence in the operated ear is connected to the pathological changes diagnosed in the opposite ear. Ilno., et al. [15] found that the dysfunction of the Eustachian tube is the most important factor for possible recidivism. Rakover., et al. [19] agree with other experts that even though the etiologic of cholesteatoma is unknown, otitis media with effusion and chronic tube dysfunction causes epithelial changes in the mucosae of middle ear, atrophy, and retraction of tympani membrane. The retraction and atrophy of the tympanic membrane can advance into adhesive otitis and may lead to cholesteatoma. Slangerup, Drozdziewicz and Tos [4] found that recurrence developed more frequently $(\mathrm{P}<$ 0.05 ) in children with poor middle ear ventilation compared those who had good middle ear ventilation (13\%). Among the high risky

Citation: Ivan Baljosevic., et al. "Potential Factors for Recurrence of Cholesteatoma in Children". Acta Scientific Orthopaedics 4.12 (2021): 53-58. 
group, the highest degree of recurrence (up to 92\%) was found in children younger than 8 , with negative Valsalva test, damage of hearing chain and extended cholesteatoma.

The highest percentage of recurrence was found in cases where otitis media with effusion has been diagnosed in the second ear ( 4 or $66 \%$ ), which could be explained by poor functioning of the Eustachian tube and poor ear ventilation. This is one of the possible causes that compromised the surgical treatment and led to the development of recurrence. Certain studies published data on the size and pneumatization of mastoid process and the degree of cholesteatoma recurrence. Hildmann and Sudhoff [20] found that chronic inflammation process causes sclerosis of mastoid cellular system. Dawes and Leaper [21] also reported that in small mastoids, it is necessary to take a more aggressive approach in completely removing the cholesteatoma during the first procedure. This includes the removal of the posterior wall of auditory canal, disarticulation of intact ossicular chain and the careful dissection of the disease around stapes and oval window. The highest level of cholesteatoma recurrence in our study (29\%), was recorded in the group with a small, poorly pneumatized mastoid process which may be attributed to poor ear ventilation.

On the end, we have established that the percentage of cholesteatoma recurrence depends on various factors: 1) type of applied surgical technique, 2) child's age, 3) extent of cholesteatoma, 4) surgeon experience, 5) possibility of monitoring the patient, 6) pneumatization of mastoid process and 7) pathological condition in the opposite ear

\section{Conclusion}

Cholesteatoma of the middle ear in children is an aggressive disease characterized by a high level of recurrences. In our studies, the highest percentage of recurrence was found during the first year after the surgery, which appeared as consequence of either the incomplete removal of cholesteatoma or the inadequate selection of surgical method.

The highest recurrence degree was diagnosed in children younger than 9, with poor middle ear ventilation, high level of hearing chain damage, pathological changes in the second ear and a poorly pneumatized mastoid.

This is a high-risk group that must be monitored for a year by the otologist.
The results obtained through the wall down surgical technique are superior, registering a lower percentage of recurrences.

The wall up technique should be used in older children, with well-pneumatized mastoid extension and cholesteatoma that does not cross over the cavum tympani. The main goal of future therapy should be the improvement of the wall down technique in terms of the prevention of retraction pocket, the decrease in residual percentage or atelectatic process and the improvement of hearing results.

\section{Bibliography}

1. Schraff SA and Strasnick B. "Pediatric cholesteatoma: A retrospective review". International Journal of Pediatric Otorhinolaryngology 70 (2006): 385-393.

2. Edelstein D., et al. "Cholesteatoma in pediatric age group". Annals of Otology, Rhinology and Laryngology 97 (1988): 23-29.

3. Stangerup S., et al. "Cholesteatoma in children, predictors and calculation of recurrence rates". International Journal of Pediatric Otorhinolaryngology 49 (1999): 69-73.

4. Stangerup S., et al. "Recurrence of attic cholesteatoma: Different methods of estimating recurrence rates". OtolaryngologyHead and Neck Surgery 123 (2000): 283-287.

5. Schmid H., et al. "Long-term results for children's cholesteatoma”. American Journal of Otolaryngology 12 (1991): 83-87.

6. Chinsky A. "Cholesteatomatous chronic otitis media". International Journal of Pediatric Otorhinolaryngology 49.1 (1999): S75-S79.

7. Shewel Y., et al. "Recidivism of childhood cholesteatoma and surgical techniques: a meta-analysis study". The Egyptian Journal of Otolaryngology 36.1 (2020): 38.

8. Khavasi P., et al. "Acquired cholesteatoma in children: presentation, complications and management". International Journal of Otorhinolaryngology and Head and Neck Surgery 4.4 (2018): 1017.

9. Soldaty D and Mudry A. "Cholesteatoma in children: techniques and results". International Journal of Pediatric Otorhinolaryngology 52 (2000): 269-276.

10. Piras G., et al. "Long term outcomes of canal wall up and canal wall down tympan mastoidectomies in pediatric cholesteatoma". International Journal of Pediatric Otorhinolaryngology (2021): 150. 
11. Darrouzet V., et al. "Preference for the Closed Technique in the Management of Cholesteatoma of the Middle Ear in Children: A Retrospective Study of 215 Consecutive Patients Treated Over 10 Years". American Journal of Otolaryngology 21.4 (2000): 474-481.

12. Vlasova GV. "Age aspects of the clinical course of the middle ear cholesteatoma in children". Vestnik Otorinolaringologii 85.1 (2020): 25-29.

13. Prasad SC., et al. "Long-term surgical and functional outcomes of the intact canal wall technique for middle ear cholesteatoma in the paediatric population". ACTA Otorhinolaryngologica Italica 34 (2014): 354-361.

14. Lima A., et al. "Is pediatric cholesteatoma more aggressive in children than in adults?" A comparative study using the EAONO/JOS classification". International Journal of Pediatric Otorhinolaryngology (2020): 138.

15. Iino Y., et al. "Hearing results of canal wall reconstruction tympanoplasty for middle ear cholesteatoma in children". International Journal of Pediatric Otorhinolaryngology 60 (2001): 65-72.

16. Roger G., et al. "Predictive risk factors of residual cholesteatoma in children: a study of 256 cases". American Journal of Otolaryngology 18 (1997): 550-558.

17. Rosenfeld RM., et al. "Predictors of residual-recurrent cholesteatomas in children". Archives of Otorhinolaryngology-Head and Neck Surgery 118 (1992): 384-391.

18. Fageeh N., et al. "Surgical Treatment of Cholesteatoma in Children". Journal of Otolaryngology 28.6 (1999): 309-312.

19. Rakover Y., et al. "Comparison of the incidence of cholesteatoma surgery before and after using ventilation tubes for secretory otitis media". International Journal of Pediatric Otorhinolaryngology 56 (2000): 41-44.

20. Hildmann H and Sudhoff H. "Cholesteatoma in children". International Journal of Pediatric Otorhinolaryngology 49 (1999): 81-86.

21. Dawes $P$ and Leaper M. "Paediatric small cavity mastoid surgery: second look tympanotomy". International Journal of Pediatric Otorhinolaryngology 68 (2004): 143-148.

Volume 4 Issue 12 December 2021

(C) All rights are reserved by Ivan Baljosevic., et al. 